\begin{tabular}{|c|c|}
\hline Title & $\begin{array}{l}\text { Indications for superselective intra arterial cisplatin infusion and concomitant radiotherapy in cases of hypopharyngeal } \\
\text { cancer }\end{array}$ \\
\hline Author(s) & $\begin{array}{l}\text { Furusawa, Jun; Homma, A kihiro; Onimaru, Rikiya; Sakashita, Tomohiro; Y oshida, Dai suke; Hatakey ama, Hiromitsu; } \\
\text { Mizumachi, Takatsugu; Kano, Satoshi; T suchiya, Kazuhiko; Y asuda, Koichi; Shirato, Hiroki; Fukuda, Satoshi }\end{array}$ \\
\hline Citation & $\begin{array}{l}\text { A uris nasus larynx, 42(6), } 443-448 \\
\text { https://doi.org/10.1016/.anl.2015.04.003 }\end{array}$ \\
\hline Issue Date & $2015-12$ \\
\hline Doc URL & http:/hdl .handle.net/2115/63714 \\
\hline Rights & $\begin{array}{l}\text { (c) 2015. This manuscript version is made available under the CC-BY-NC-ND } 4.0 \text { license } \\
\text { http://creativecommons.org/icenses/by-nc-nd/4.0/ }\end{array}$ \\
\hline Rights(URL) & http://creativecommons.org/icenses/by-nc-nd/4.0/ \\
\hline Type & article (author version) \\
\hline File Information & manuscript.pdf \\
\hline
\end{tabular}

Instructions for use 


\section{Indications for superselective intra-arterial cisplatin infusion and concomitant radiotherapy in cases of hypopharyngeal cancer}

Jun Furusawa ${ }^{a}$, Akihiro Homma ${ }^{a}$, Rikiya Onimaru ${ }^{b}$, Tomohiro Sakashita ${ }^{a}$, Daisuke Yoshida, Hiromitsu Hatakeyama ${ }^{a}$, Takatsugu Mizumachi ${ }^{a}$, Satoshi Kano ${ }^{a}$, Kazuhiko Tsuchiya ${ }^{b}$, Koichi Yasuda ${ }^{b}$, Hiroki Shirato ${ }^{\mathrm{b}}$, Satoshi Fukuda ${ }^{\mathrm{a}}$

a Department of Otolaryngology-Head \& Neck Surgery, Hokkaido University Graduate School of Medicine, Kita 15, Nishi 7, Kita-ku, Sapporo 060-8638, Japan

${ }^{b}$ Department of Radiology, Hokkaido University Graduate School of Medicine, Kita 15, Nishi 7, Kita-ku, Sapporo 060-8638, Japan

Corresponding author: Akihiro Homma <ak-homma@med.hokudai.ac.jp>

Department of Otolaryngology-Head and Neck Surgery, Hokkaido University Graduate School of Medicine, Kita 15, Nishi 7, Kita-ku, Sapporo 060-8638, Japan. Tel.: +81 11707 3387; fax: +81 11717 7566. 


\section{ABSTRACT}

Objective: We retrospectively assessed the indications for superselective intra-arterial infusion of cisplatin with concomitant radiotherapy (RADPLAT) in patients with hypopharyngeal cancer (HPC).

Methods: Between April 2000 and March 2013, 41 previously untreated patients received superselective intra-arterial infusion of cisplatin $\left(100-120 \mathrm{mg} / \mathrm{m}^{2}\right.$ per week) with simultaneous intravenous infusions of thiosulfate to neutralize cisplatin toxicity and conventional radiotherapy (65-70Gy).

Results: During the median follow-up period of 5.5 years, a statistically significant difference in the 5 -year overall survival was noted between patients with N0-1 $(n=14)$ and N2b-3 disease $(n=27)$. One-half of deaths were observed to be the result of distant metastasis. The 5-year local control and overall survival were significantly better in patients with unilateral than in those with bilateral primary tumors. All patients with $\mathrm{T} 4 \mathrm{~b}$ disease $(\mathrm{n}=3)$ died of disease within two years.

Conclusion: Indications for RADPLAT in patients with HPC were defined as patients with unilateral tumors staged as T3-4a and N0-1. 


\section{Introduction}

RADPLAT, superselective intra-arterial infusion of cisplatin with concomitant radiotherapy, was first reported by Robbins et al. [1] and has been shown to be a promising treatment [2,3], achieving a 90\% complete response rate in cases of advanced head and neck cancer. With regard to hypopharyngeal cancer (HPC), Samant et al. reported that the 5-year overall survival and locoregional control rates were around $23 \%$ and $90 \%$, respectively [4]. Nomura et al. also reported that the 3 -year overall survival and local control rates were around 52.2\% and 81.6\%, respectively [5]. However, a group in the Netherlands reported a multicenter, randomized phase 3 trial, in which HPC was performed for 19\% of patients in the intra-arterial group and $18 \%$ of those in the intravenous group, and concluded that intra-arterial chemoradiation was not superior to intravenous chemoradiation [6]. However, as several points in association with this trial remain controversial [5,7], we decided to retrospectively examine the indications for RADPLAT, with a particular focus on TN stage, in patients with HPC.

\section{Materials and Methods}

Eligibility criteria. From April 2000 through March 2013, 41 previously untreated patients with HPC were treated at Hokkaido University Hospital (Sapporo, Japan). Details of our RADPLAT protocol have been published elsewhere [3]. Briefly, all patients were initially evaluated by a multidisciplinary team consisting of otolaryngologists, radiation oncologists and medical oncologists, and tumors were classified according to the 7th Edition of the Union for International Cancer Control (UICC) staging system. The stage of the tumor was determined on the basis of patient history, physical examination, chest x-rays, MRI, and whole body CT or FDG-PET-CT. All the patients either had disease for which radical surgery was contraindicated or had rejected radical surgery. Unresectable disease was defined as stage T4b according to the 7th Edition of UICC staging system for primary sites or disease in which neck lymph node metastases encased the common or internal carotid artery or invaded the prevertebral fascia. Medical unsuitability for resection and refusal of surgery were not included in the definition of unresectable disease. The remaining eligibility criteria for treatment in this study included an absence of evidence of distant metastases, World Health Organization performance status of 0 to 2 , age $\leq 65$, adequate $\mathrm{renal} /$ hematologic function, and informed consent.

Chemotherapy. All patients received concurrent intra-arterial cisplatin $\left(100-120 \mathrm{mg} / \mathrm{m}^{2}\right.$ per week) and intra-venous sodium thiosulfate infusions (approximately $24 \mathrm{~g}$ ) as described in our previous report [7]. In order to evaluate the distribution of the arterial supply in the primary tumor and metastatic neck nodes, we used Digital Subtraction Angiography (DSA). With regard to the latest 32 patients, we used CT-angiography as well as DSA so as to be able to evaluate the distribution more precisely. After the feeding vessels that encompassed the targeted tumor had been identified by staining during the DSA and/or the enhancement of selected vessels during the CT-angiography, cisplatin was infused through a 
microcatheter placed in the artery. When several arteries required infusion, the dose of cisplatin for each artery was determined by the percentage of the tumor enhanced with DSA and/or CT for each intra-arterial infusion. In patients with nodes greater than $3 \mathrm{~cm}$, a portion of the cisplatin (approximately 20-30mg) was delivered to this region if fed by an artery. Chemotherapy was repeated three to four times and completed during the first 4-5 weeks.

Radiotherapy. All patients received external-beam radiotherapy to the primary sites and regional lymphatic area as described in our report [8]. Until May 2006, the irradiation schedule was 66 Gy in 33 fractions over 6.5 weeks. After May 2006, it was changed to 70Gy in 35 fractions over 7 weeks for all patients with advanced head and neck cancer. Therefore, 23 patients received 66Gy and 18 patients received 70Gy. This treatment was administered to all patients, except for one patient who received intensity-modulated radiotherapy (IMRT) as IMRT was not covered by health insurance in Japan for use on HPC at that time.

Management of the neck. Patients with regional lymph node metastasis of the neck were treated with 66-70 Gy of radiotherapy and chemotherapy. If lymph node metastases remained or recurred, patients with resectable neck disease were referred for neck dissection.

Management of the primary lesion, evaluation of response and toxicity. Responses were evaluated by clinical examination, together with $\mathrm{CT}$ and/or MRI studies at 6-8 weeks after the completion of therapy. As it is difficult to differentiate between radiographic changes related to the treatment and scar tissue from persisting tumors, this uncertainty is reflected in the treatment response. Over time, scar tissue remains stable, but persistent tumor tissue will progress, so a patient with radiologic changes that remained stable and with no signs or symptoms of disease was considered to be progression free [3]. Based on this concept, we adopted a 'wait-and-see' policy for the primary lesion, even if we could detect the presence of residual tumor tissue. A biopsy was performed only when indicated to document recurrence. All toxicities encountered during therapy were evaluated according to the Common Terminology Criteria for Adverse Events v4.0 (2010).

Statistical Analysis. The primary endpoint of the study was overall survival and the second endpoints were local control and toxicity. All patients were closely observed during the follow-up period, the median length of which was 5.5 years (range 1.2-8.7 years).

Cases of progression and with signs or symptoms of primary disease after the completion of RADPLAT were considered to be local failures, regardless of whether salvage was successful. Overall survival, which included death from any cause, and local control probabilities, computed from the beginning of treatment to the time of local failure, were calculated by the Kaplan-Meier method and 
compared using the log-rank test. The level of statistical significance was set at $P<0.05$. Statistical analysis was performed using JMP Pro 11.0.0 statistical software (SAS Institute, Cary, NC, USA).

\section{Results}

Patient characteristics. Forty-one patients were enrolled in this study and evaluation was possible in all cases. Patient details are given in Table 1. All tumors were determined histologically to be squamous cell carcinoma. $\mathrm{T}$ and $\mathrm{N}$ classifications are shown in Table 2. Induction chemotherapy was performed for 3 patients. One patient with T4aN2b pyriform sinus cancer received one course of CDDP and 5-FU-based chemotherapy. The remaining 2 patients received Docetaxel, CDDP and 5-FU-based chemotherapy: one patient with T4aN0 pyriform sinus cancer received one course and the other patient with T4bN3 pyriform sinus cancer received 2 courses.

Compliance. RADPLAT was feasible (three or four infusions of intra-arterial cisplatin and a full dose of radiation therapy within 7 days of treatment interruptions) in 38 patients (92.7\%). One patient received two infusions of intra-arterial chemotherapy because of heart disease. Two patients did not complete radiotherapy: one patient with $\mathrm{T} 4 \mathrm{aN} 3$ pyriform sinus cancer stopped radiotherapy after four intra-arterial infusions and 40Gy of radiotherapy because of a distant metastasis that worsened rapidly, and another patient with T4bN3 pyriform sinus cancer stopped after four intra-arterial infusions and 54.5Gy of radiotherapy because of quadriplegia resulting from intra-arterial infusion.

Toxicity. Although the treatment regimen was intensive, acute toxicity was manageable in most patients. The details are given in Table 3. No patient died as a result of treatment toxicity, although 33 patients (80.5\%) experienced grade III-IV toxicity. One patient experienced a neurologic sequela (quadriplegia) [3], although he subsequently recovered use of his upper, but not his lower extremities. One patient with T4aN0 pyriform sinus cancer that extended inferiorly toward the esophagus needed feeding-tube support after therapy due to pharyngeal stenosis, but none of the other surviving patients required feeding-tube support after therapy. No osteonecrosis, chondronecrosis or mucosal ulceration was observed as late adverse reactions.

Overall survival and local control rates. The 5 -year overall survival was $51.3 \%$, and the 5 -year local control was $81.6 \%$ for all patients (Figure 1). The 5 -year overall survival was $83.9 \%$ for patients with N0-1 disease $(n=14)$, which was significantly $(\mathrm{p}=0.0029)$ better than that for patients with $\mathrm{N} 2 \mathrm{~b}-3$ disease (35.4 \%, $\mathrm{n}=27$ ) (Figure 2). The 5-year local control was $90.5 \%$ for patients with T2-3 disease $(\mathrm{n}=22)$ and $80.0 \%$ for those with T4a disease $(\mathrm{n}=16)$, and no statistically significant difference was observed between the groups $(\mathrm{p}=0.36$ ) (Figure $3 \mathrm{~A})$. We defined a "unilateral primary tumor" as not extending beyond the midline of the posterior wall, whereas a "bilateral primary tumor" extended beyond the 
midline of posterior wall. The 5 -year local control was $95.7 \%$ for patients with a unilateral primary tumor, which was significantly $(\mathrm{p}=0.0048)$ better than that for patients with a bilateral tumor (60.4\%) (Figure 3B). With regard to overall survival, the 5-year overall survival was $48.9 \%$ for patients with T2-3 disease and $62.5 \%$ for those with T4a disease (Figure 4A). The 5-year overall survival was $74.5 \%$ for patients with a unilateral primary tumor, which was significantly better than that for those with a bilateral tumor $(22.2 \%)(\mathrm{p}<0.0001$, Figure $4 \mathrm{~B})$.

Primary disease response. Of the 41 patients enrolled in the treatment program, a complete response at the primary site was obtained in $34(82.9 \%)$ and a partial response in $7(17.1 \%)$ patients. The primary disease was well controlled by RADPLAT in 34 patients (82.9\%). The remaining 7 patients (17.1\%) showed progression and with signs or symptoms of primary disease after the completion of RADPLAT. Of these, two patients did not complete radiotherapy for the reasons mentioned above. The subsite for each of the other 3 patients was the posterior wall.

Neck disease response. Eight patients negative for neck disease were well controlled (Figure 5). Among the 33 patients with positive necks, a complete response was obtained in 17 (51.5\%) and a partial response in 16 (48.5\%) patients. Twenty-three patients were well controlled without surgery until the final follow-up. Seven of nine patients with uncontrolled neck disease underwent neck dissection for persistent and recurrent neck disease after treatment. The remaining two patients were not indicated for salvage neck dissection as their neck diseases were accompanied by unresectable primary disease. In spite of no neck recurrence, one patient with $\mathrm{N} 1$ disease received simultaneous neck dissection during reconstruction surgery for recurrent primary disease.

Pattern of relapse. The site of first failure was identified wherever possible. Failure first occurred at the primary site in 7 patients. Salvage surgery was indicated for only one patient with T4aN1 pyriform sinus cancer, who underwent surgery involving total laryngectomy and pharyngectomy, but developed distant metastasis within the first year post-surgery. The remaining 6 patients were not indicated for radical surgery, and died of primary disease. Failure first occurred at the neck in 7 patients. As a result, 5 of the 7 patients were treated successfully by salvage neck dissection, and 2 of these 5 patients developed distant metastasis after surgery. The remaining 2 patients had recurrent neck disease after surgery, of which they later died. Distant metastasis was found in 7 patients without primary or neck recurrence. In total, 10 of 20 deaths were associated with distant metastasis, and all had an N stage of N2b-3, except for one patient with N1. Two patients died of causes other than disease.

Tracheostomy. Five patients underwent tracheostomy prior to RADPLAT due to airway compromise, and one patient required a delayed tracheostomy due to radiation injury presenting as persistent stridor 
and laryngeal edema. Four of these patients were successfully decannulated shortly after the completion of treatment. Two patients could not be decannulated because of persistent or recurrent primary or distant disease.

\section{Discussion}

First, there are several points of controversy with regard to the randomized trial conducted by the group in the Netherlands [6] that must be discussed. As mentioned elsewhere, their report made no mention of the angiographic technique used or the adequacy of cisplatin infusion into the tumor-feeding artery [7]. Further, HPC patients comprised only a small subgroup of the total study population, and this trial targeted unresectable patients, which meant that it could not assess organ preservation [5]. In addition, 62-64\% of patients had oropharyngeal cancer (OPC), and it has been reported that approximately $60 \%$ or more of OPCs are HPV related [9]. This means that these subgroups were very sensitive to chemotherapy, regardless of whether it was intra-arterial or intravenous. Finally, since RADPLAT is a time-consuming and complicated treatment, it may be difficult to apply RADPLAT in all cases of head and neck cancer. For these reasons, we decided to investigate the indications for RADPLAT with a focus on cases of HPC, and sought the appropriate indications only after consideration of the characteristics of HPC, such as arterial blood supply and rich lymphatics, that result in a high incidence of lymph node metastases.

To seek the appropriate indications for HPC, we first considered $\mathrm{T}$ stage. As mentioned previously, conventional treatments, such as extensive surgery, have a negative impact on the patient's quality of life. Radiotherapy alone may be effective for the treatment of cases at an early $\mathrm{T}$ stage [10], but not those at an advanced $\mathrm{T}$ stage [11]. Urba et al. reported on the efficacy of combination therapy, consisting of systemic chemotherapy followed by radiotherapy, but excluded T4 tumors [12]. T4a stage, which is usually associated with significant cartilage destruction prior to treatment, is usually indicated for radical surgery, such as laryngectomy, as the cartilage destruction limits the likelihood of an adequate response and functional recovery after treatment with larynx-preserving approaches, and increases the probability of radiation-induced necrosis [13, 14]. In our study, results showed an absence of any radiation-induced necrosis of the laryngeal cartilage. Further, we did not detect any significant difference between T1-3 and T4a stage in terms of local control and overall survival (Figure 3A, 4A). In addition, significantly better local control and overall survival were observed for unilateral than for bilateral tumors (Figure 3B, 4B). Similarly, it has been reported that unilateral infusion was superior to bilateral infusion in an unplanned subgroup analysis $[5,15]$. This indicates that the laterality of the tumor has an impact on local control and overall survival. Considering these results, we speculated that arterial supply to the hypopharynx is complicated [16], particularly as the posterior wall of the hypopharynx is supplied by the ascending pharyngeal artery, inferior thyroid artery, and lingual artery as well as the superior thyroid artery. Hence, the intra-arterial infusion of cisplatin may not cover the entire primary tumor. As a 
consequence, we consider the indication for RADPLAT in terms of T stage to be unilateral T3 or 4a. It goes without saying that the T4b tumors were difficult to control, and all patients with T4b disease died of disease within two years (Figure 3A). On the other hand, tumors at an early $\mathrm{T}$ stage may be controlled by radiotherapy alone, systemic concurrent chemoradiotherapy or partial pharyngectomy.

Next, we considered the N stage. Where possible, the risk of occult lymph node metastases or residual disease following treatment should be controlled by neck dissection. Meanwhile, patients with advanced $\mathrm{N}$ stage are at high-risk for distant metastases [17-19], and this had an impact on our results. We observed a significant difference in overall survival between patients with N0-1 stage and those with N2b-3 stage (Figure 2). Further, one-half of all deaths were related to distant metastasis, with the N stage of the patients who died being N2b-3, except for one patient with N1. Similarly, distant metastasis has been reported to be involved in 40\% [4] and 39\% [5] of failed cases. These results suggest that the indication for RADPLAT in terms of $\mathrm{N}$ stage is below N2. RADPLAT, as well as concurrent chemoradiotherapy, may be less effective in preventing the development of distant metastases in comparison with induction chemotherapy [20]. In other words, stage N2 or higher is a good indication for sequential therapy; i.e., induction chemotherapy followed by concurrent chemoradiotherapy. A three-drug combination in which taxane is added to a cisplatin plus 5-fluorouracil regimen significantly improved the response rate as well as overall survival compared with cisplatin plus 5-fluorouracil alone [21]. We speculate that, after 2-3 courses of induction chemotherapy, most of the primary tumors should have been resolved and can, thereafter, be controlled by systemic concurrent chemoradiotherapy.

Concurrent chemoradiotherapy is associated with a higher rate of treatment-related and unexplained deaths [11]; however, we encountered no treatment-related or unexplained deaths in our study. Intra-arterial chemotherapy, however, does involve a risk of catheter-related problems. We experienced one case of hypoglossal nerve paralysis with full recovery and another case of quadriplegia. The later patient subsequently recovered use of the upper, but not of the lower extremities. We speculated that the thyrocervical trunk, from which the cisplatin was infused, communicates with the anterior spinal artery. Apart from these events, all other toxicities, episodes of mucositis and dermatitis, decreases in white blood cell count and other complications were regarded as manageable and were well controlled, though approximately $80 \%$ of patients experienced grade III-IV toxicity. Therefore, we obtained good compliance with this treatment regimen. The RADPLAT protocol exposes the tumor and surrounding normal tissues to an extraordinarily high concentration of cisplatin, and this increased concentration of cisplatin within the local tissues of the head and neck might result in some swallowing dysfunction. Furthermore, at higher T stages, patients are more likely to have swallowing difficulty [22]. Although our study included 14 patients with T3 and 16 patients with T4a primary tumors, all of the no tumor-bearing patients were able to achieve oral intake without feeding-tube support either before or after treatment, except for one patient with T4a pyriform sinus cancer. Regarding this one case, the primary tumor, derived from the pyriform sinus, extended inferiorly toward the esophagus, leading to a swallowing 
disorder before treatment. We speculated that this symptom would worsen after treatment, but the patient preferred to preserve the larynx. Eventually, feeding-tube support was needed, but the larynx and speaking ability were preserved and the patient is satisfied with the current situation. Of the 41 patients, 6 received tracheostomy, with 4 of the 6 successfully decannulated shortly after the completion of RADPLAT unlike a previous study in which all patients underwent tracheostomy before the treatment [5]. We speculate that tracheostomy is, therefore, not indispensable for RADPLAT.

\section{Conclusions}

Indications for RADPLAT in patients with HPC were defined as a T stage of T3-4a in unilateral cases (not extending beyond the midline of the posterior wall) and an $\mathrm{N}$ stage of N0-1. Our retrospective study revealed that the toxicities were manageable and no treatment-related or unexplained deaths were encountered. We concluded that RADPLAT for HPC is effective if these indications are taken into consideration. Therefore, more detailed randomized trials comparing the results for RADPLAT with those for systemic chemoradiotherapy are expected in the future.

\section{Acknowledgments}

This study was supported in part by Health and Labour Sciences Research Grants for Clinical Cancer Research (H22-017 and H26-141) from the Ministry of Health, Labour and Welfare of Japan, the National Cancer Center Research and Development Fund (23-A-21 and 26-A-4) of Japan, and a Grant-in-Aid for Scientific Research (C) (KAKENHI 24592587) from the Ministry of Education, Culture, Sports, Science, and Technology of Japan.

\section{Financial support}

None.

\section{Conflict of interest}

None. 


\section{References}

[1] Robbins KT, Vicario D, Seagren S, Weisman R, Pellitteri P, Kerber C, et al. A targeted supradose cisplatin chemoradiation protocol for advanced head and neck cancer. Am J Surg. 1994;168:419-422. [2] Robbins KT, Kumar P, Wong FS, Hartsell WF, Flick P, Palmer R, et al. Targeted chemoradiation for advanced head and neck cancer: analysis of 213 patients. Head Neck. 2000;22:687-693.

[3] Homma A, Furuta Y, Suzuki F, Oridate N, Hatakeyama H, Nagahashi T, et al. Rapid superselective high-dose cisplatin infusion with concomitant radiotherapy for advanced head and neck cancer. Head Neck. 2005;27:65-71.

[4] Samant S, Kumar P, Wan J, Hanchett C, Vieira F, Murry T, et al. Concomitant radiation therapy and targeted cisplatin chemotherapy for the treatment of advanced pyriform sinus carcinoma: disease control and preservation of organ function. Head Neck. 1999;21:595-601.

[5] Nomura K, Takahara M, Katayama A, Kishibe K, Kunibe I, Katada A, et al. Intra-arterial cisplatin with concomitant radiation for advanced hypopharyngeal cancer. Laryngoscope. 2013;123:916-922.

[6] Rasch CR, Hauptmann M, Schornagel J, Balm AJ, Hilgers FJ. Intra-arterial Versus intravenous chemoradiation for advanced head and neck cancer: Results of a randomized phase 3 trial. Cancer. 2010;116:2159-2165.

[7] Homma A, Sakashita T, Yoshida D, Onimaru R, Tsuchiya K, Suzuki F, et al. Superselective intra-arterial cisplatin infusion and concomitant radiotherapy for maxillary sinus cancer. Br J Cancer. 2013;109:2980-2986.

[8] Taki S, Homma A, Suzuki F, Oridate N, Hatakeyama H, Mizumachi T, et al. Combined modality therapy for laryngeal cancer with superselective intra-arterial cisplatin infusion and concomitant radiotherapy.

Int J Clin Oncol. 2012;17:441-446.

[9] Mehanna H, Beech T, Nicholson T, El-Hariry I, McConkey C, Paleri V, et al. Prevalence of human papillomavirus in oropharyngeal and nonoropharyngeal head and neck cancer--systematic review and meta-analysis of trends by time and region. Head Neck. 2013;35:747-755.

[10] Foote RL. Radiotherapy alone for early-stage squamous cell carcinoma of the larynx and hypopharynx. Int J Radiat Oncol Biol Phys. 2007;69(2 Suppl):S31-36.

[11] Forastiere AA, Zhang Q, Weber RS, Maor MH, Goepfert H, Pajak TF, et al. Long-term results of RTOG 91-11: a comparison of three nonsurgical treatment strategies to preserve the larynx in patients with locally advanced larynx cancer. J Clin Oncol. 2013;31:845-852.

[12] Urba SG, Moon J, Giri PG, Adelstein DJ, Hanna E, Yoo GH, et al. Organ preservation for advanced resectable cancer of the base of tongue and hypopharynx: a Southwest Oncology Group Trial. J Clin Oncol. 2005;23:88-95. 
[13] The Department of Veterans Affairs Laryngeal Cancer Study Group. Induction chemotherapy plus radiation compared with surgery plus radiation in patients with advanced laryngeal cancer. N Engl J Med. 1991;324:1685-1690.

[14] Becker M. Neoplastic invasion of laryngeal cartilage: radiologic diagnosis and therapeutic implications. Eur J Radiol. 2000;33:216-229.

[15] van den Broek GB, Rasch CR, Pameijer FA, Peter E, van den Brekel MW, Tan IB, et al. Pretreatment probability model for predicting outcome after intraarterial chemoradiation for advanced head and neck carcinoma. Cancer. 2004;101:1809-1817.

[16] Terayama N, Sanada J, Matsui O, Kobayashi S, Kawashima H, Yamashiro M, et al. Feeding artery of laryngeal and hypopharyngeal cancers: role of the superior thyroid artery in superselective intraarterial chemotherapy. Cardiovasc Intervent Radiol. 2006;29:536-543.

[17] León X, Quer M, Orús C, del Prado Venegas M, López M. Distant metastases in head and neck cancer patients who achieved loco-regional control. Head Neck. 2000;22:680-686.

[18] Spector JG, Sessions DG, Haughey BH, Chao KS, Simpson J, El Mofty S, et al. Delayed regional metastases, distant metastases, and second primary malignancies in squamous cell carcinomas of the larynx and hypopharynx. Laryngoscope. 2001;111:1079-1087.

[19] Garavello W, Ciardo A, Spreafico R, Gaini RM. Risk factors for distant metastases in head and neck squamous cell carcinoma. Arch Otolaryngol Head Neck Surg. 2006;132:762-766.

[20] Vokes EE. Competing roads to larynx preservation. J Clin Oncol. 2013;31:833-835.

[21] Vermorken JB, Remenar E, van Herpen C, Gorlia T, Mesia R, Degardin M, et al. Cisplatin, fluorouracil, and docetaxel in unresectable head and neck cancer. N Engl J Med. 2007;357:1695-1704.

[22] Pauloski BR, Rademaker AW, Logemann JA, Stein D, Beery Q, Newman L, et al. Pretreatment swallowing function in patients with head and neck cancer. Head Neck. 2000;22:474-482. 


\section{Legend}

Figure 1. Overall survival and Local control $(n=41)$

Figure 2. Overall survival according to $\mathrm{N}$ classification

Figure 3A. Local control according to T classification

Figure 3B. Local control (Unilateral versus Bilateral)

Figure 4A. Overall survival according to $\mathrm{T}$ classification

Figure 4B. Overall survival (Unilateral versus Bilateral)

Figure 5. Response to RADPLAT: Neck disease

*One patient without neck recurrence received simultaneous neck dissection during reconstruction surgery for recurrent primary disease.

**Two patients were not indicated for salvage neck dissection as their neck diseases were accompanied by unresectable primary disease. 
Table 1 Clinical characteristics $(n=41)$

Sex

Male

36

Female

5

Age (years)

Range

45-74

Median

59

Mean

58.6

Operability

Unresectable

11

Resectable

30

Tumor subsite

$\begin{array}{ll}\text { pyriform sinus } & 35 \\ \text { posterior wall } & 5 \\ \text { postcricoid } & 1\end{array}$

35

5 
Table $2 \mathrm{~T}$ and $\mathrm{N}$ stage $(\mathrm{n}=41)$

\begin{tabular}{|l|c|c|c|c|c|c|c|}
\hline \multicolumn{7}{|l|}{ No. of patients by N classification } \\
\hline T classification & 0 & 1 & $2 \mathrm{a}$ & $2 \mathrm{~b}$ & $2 \mathrm{c}$ & 3 & Total \\
\hline 2 & 3 & 2 & & 3 & & & 8 \\
\hline 3 & 1 & 2 & & 8 & 2 & 1 & 14 \\
\hline $4 \mathrm{a}$ & 4 & 2 & & 7 & 2 & 1 & 16 \\
\hline $4 \mathrm{~b}$ & 8 & 6 & 0 & 18 & 5 & 4 & 41 \\
\hline Total & & & & & 1 & 2 & 3 \\
\hline
\end{tabular}


Table 3 Acute Toxicity $(n=41)$

\begin{tabular}{|l|c|c|c|c|}
\hline & \multicolumn{5}{|c|}{ No. of patients by toxicity grade } \\
\hline Toxicity & I & II & III & IV \\
\hline Anemia & 17 & 16 & 6 & \\
\hline Leukoplakia & 3 & 19 & 14 & \\
\hline Thrombocytopenia & 12 & 1 & 6 & 1 \\
\hline Fever & 16 & 5 & 2 & \\
\hline Weight loss & 17 & 8 & & \\
\hline Dermatitis & 5 & 25 & 10 & \\
\hline Nause/Vomiting & 19 & 7 & 7 & \\
\hline Mucositis & 2 & 21 & 16 & \\
\hline Liver dysfunction & 17 & 2 & 1 & \\
\hline Renal & 2 & 1 & & \\
\hline Hyperglycemia & 6 & 3 & 2 & 1 \\
\hline Hyperkalemia & & & 1 & \\
\hline Thromboembolic event & & & 2 & \\
\hline Hypoglossal nerve disorder & 1 & & & \\
\hline Nervous system disorders & & & & 1 \\
\hline
\end{tabular}


Fig. 1 Overall survival and Local control $(n=41)$

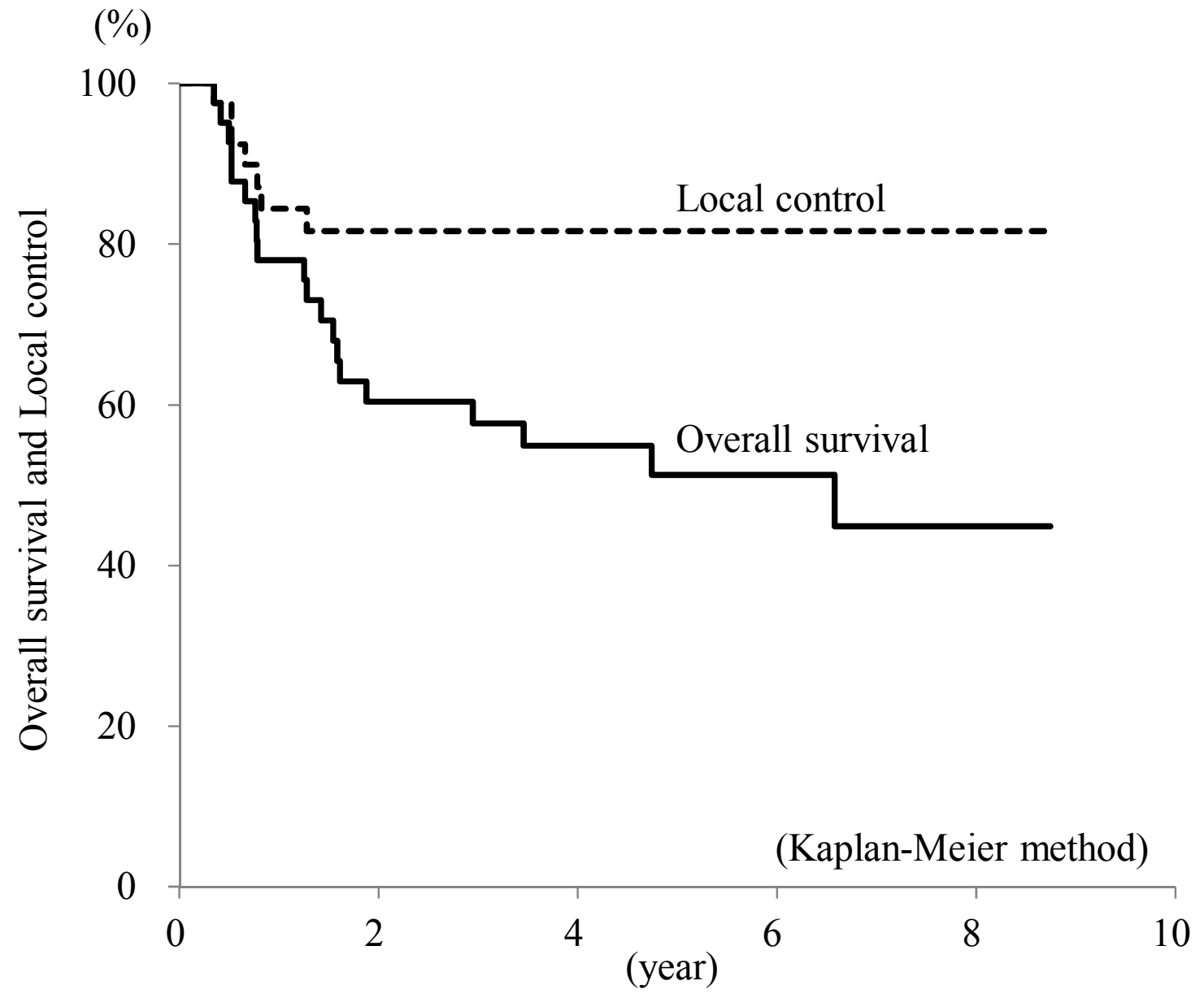

Patients at risk

$\begin{array}{llllllllll}\text { OS } & 41 & 32 & 24 & 21 & 19 & 13 & 10 & 7 & 3 \\ \text { LC } & 41 & 31 & 24 & 21 & 19 & 13 & 10 & 7 & 3\end{array}$


Fig. 2 Overall survival according to $\mathrm{N}$ classification

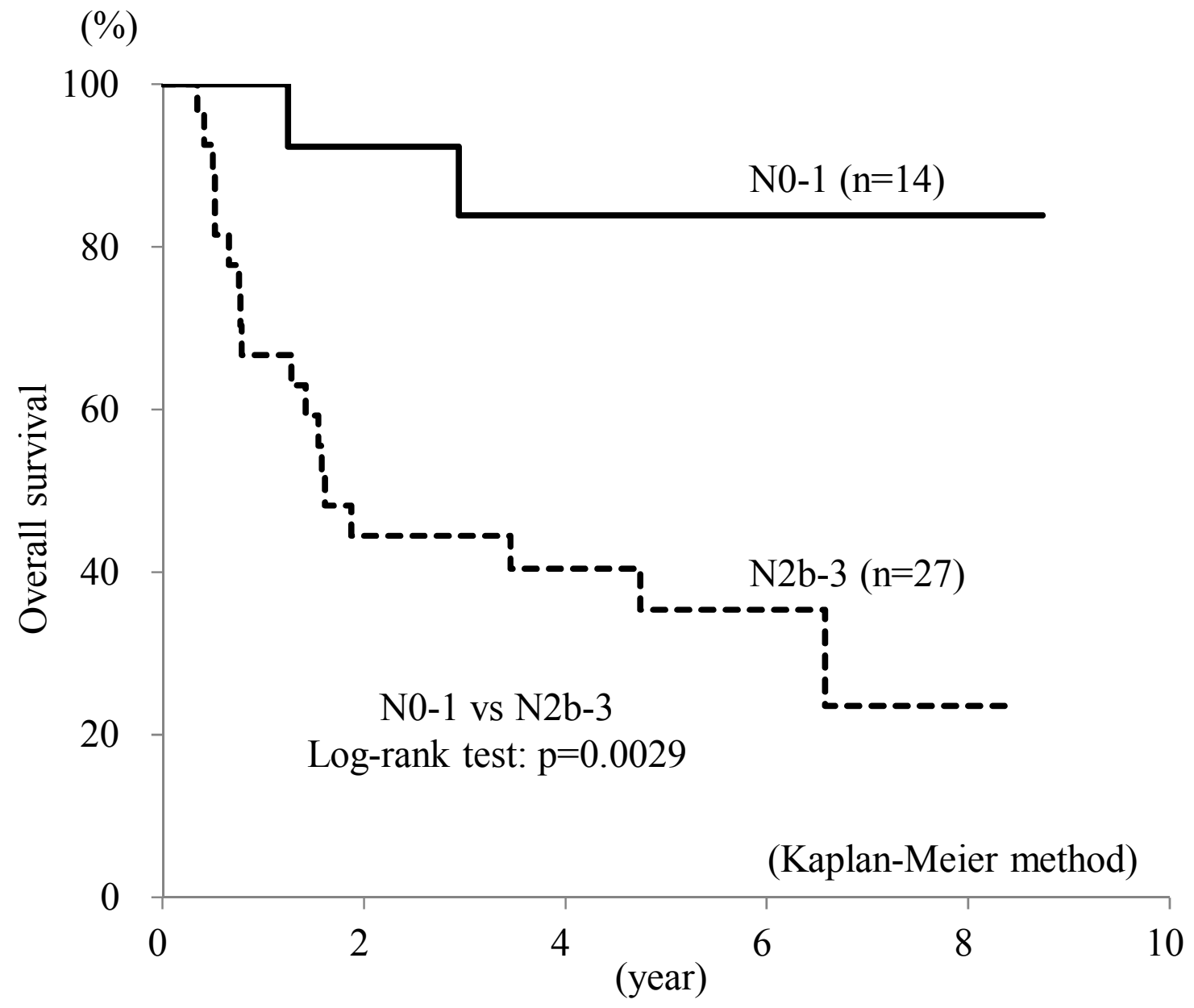

Patients at risk

$\begin{array}{lrrrrrrrrr}\text { N0-1 } & 14 & 14 & 12 & 10 & 10 & 7 & 5 & 5 & 2 \\ \text { N2b-3 } & 27 & 16 & 12 & 11 & 9 & 6 & 5 & 2 & 1\end{array}$


Fig. 3A Local control according to T classification

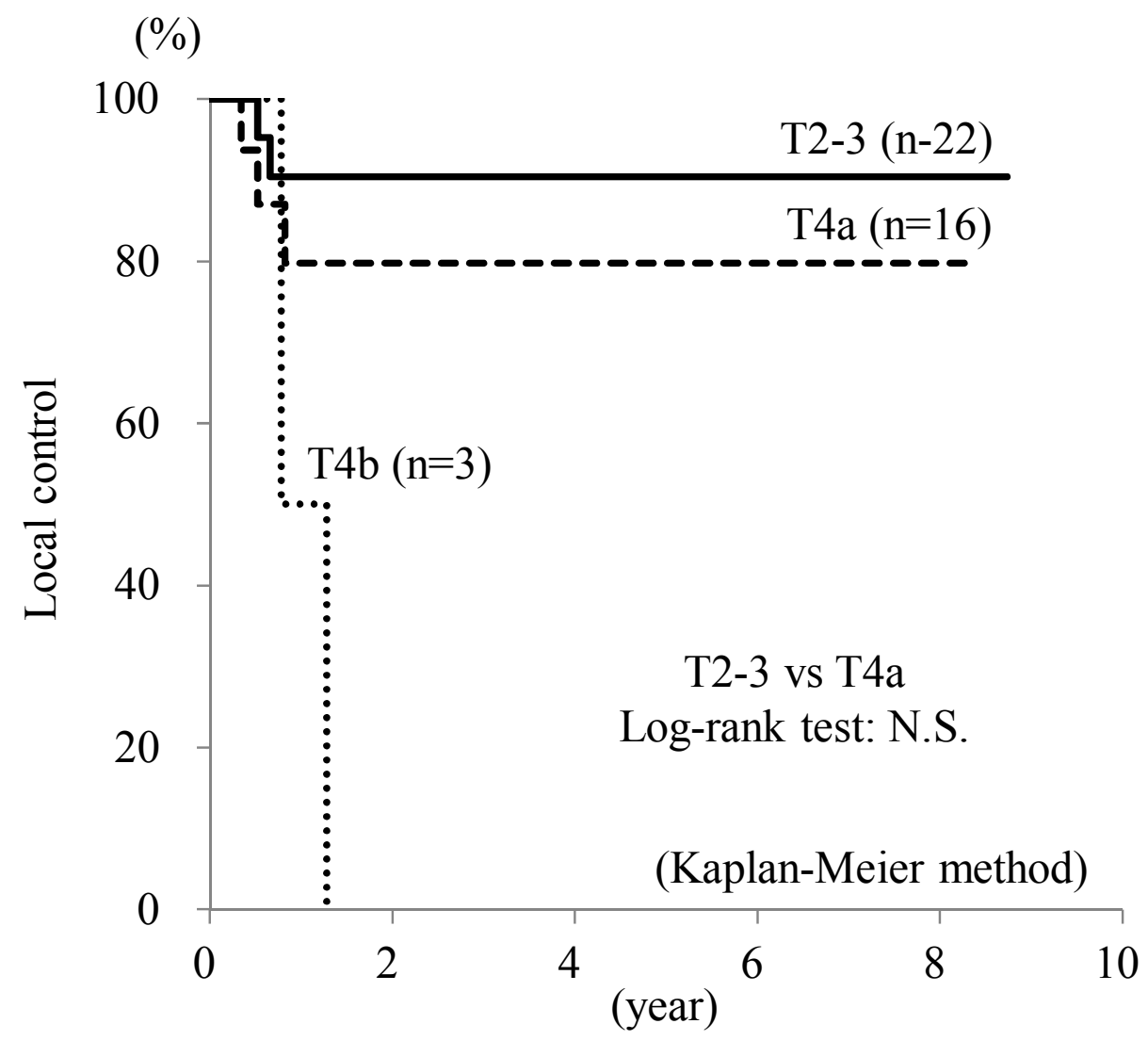

Patients at risk

$\begin{array}{lrrrrrrrrr}\text { T2-3 } & 22 & 19 & 14 & 11 & 10 & 7 & 7 & 4 & 1 \\ \text { T4a } & 16 & 11 & 10 & 10 & 9 & 6 & 3 & 3 & 2 \\ \text { T4b } & 3 & 1 & & & & & & & \end{array}$

Fig. 3B Local control (Unilateral versus Bilateral)

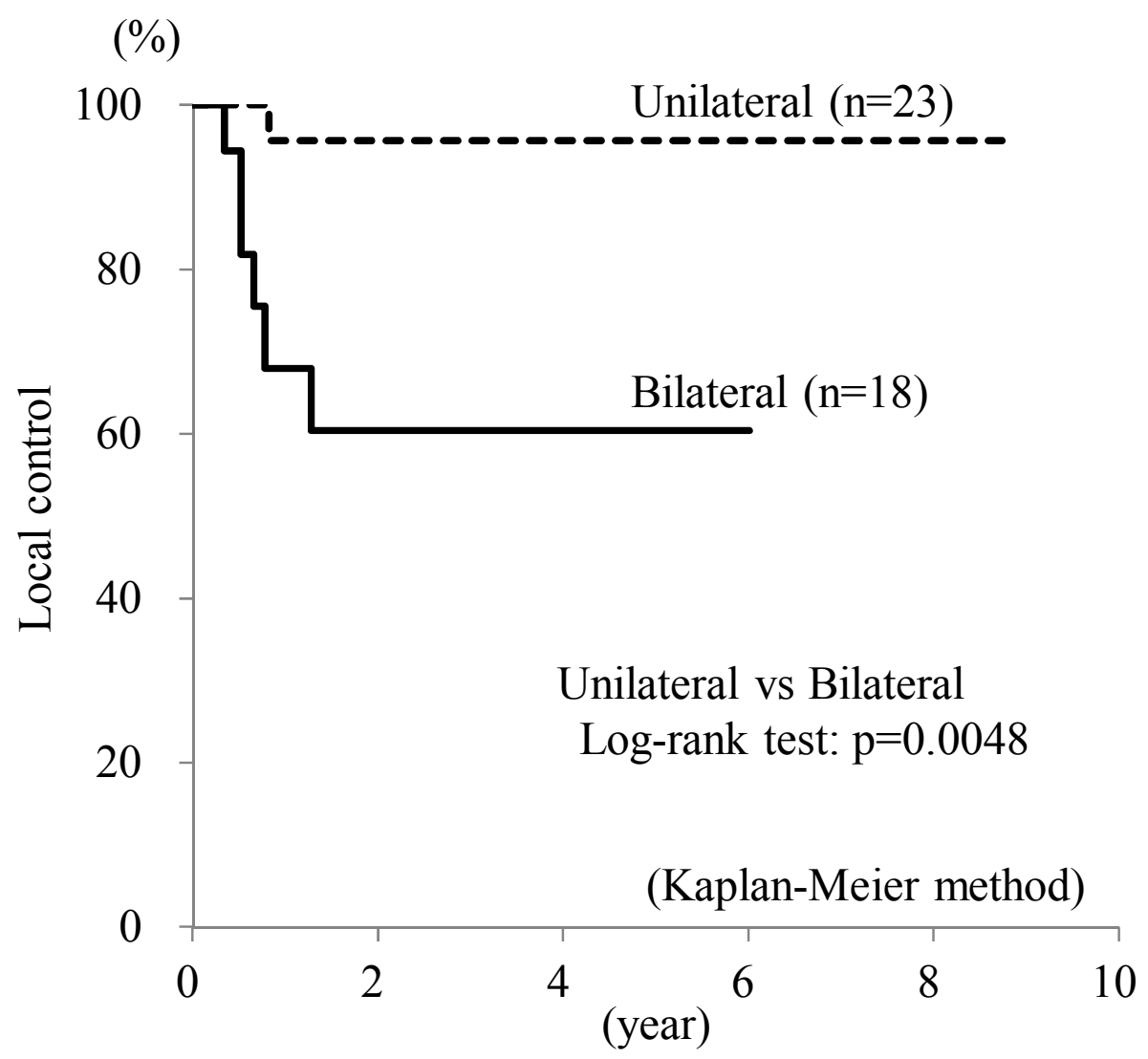

Patients at risk

$\begin{array}{llllllllll}\text { Unilateral } & 23 & 22 & 19 & 16 & 15 & 10 & 9 & 7 & 3\end{array}$

$\begin{array}{llllllll}\text { Bilateral } & 18 & 9 & 5 & 5 & 4 & 3 & 1\end{array}$ 
Fig. 4A Overall survival according to $T$ classification

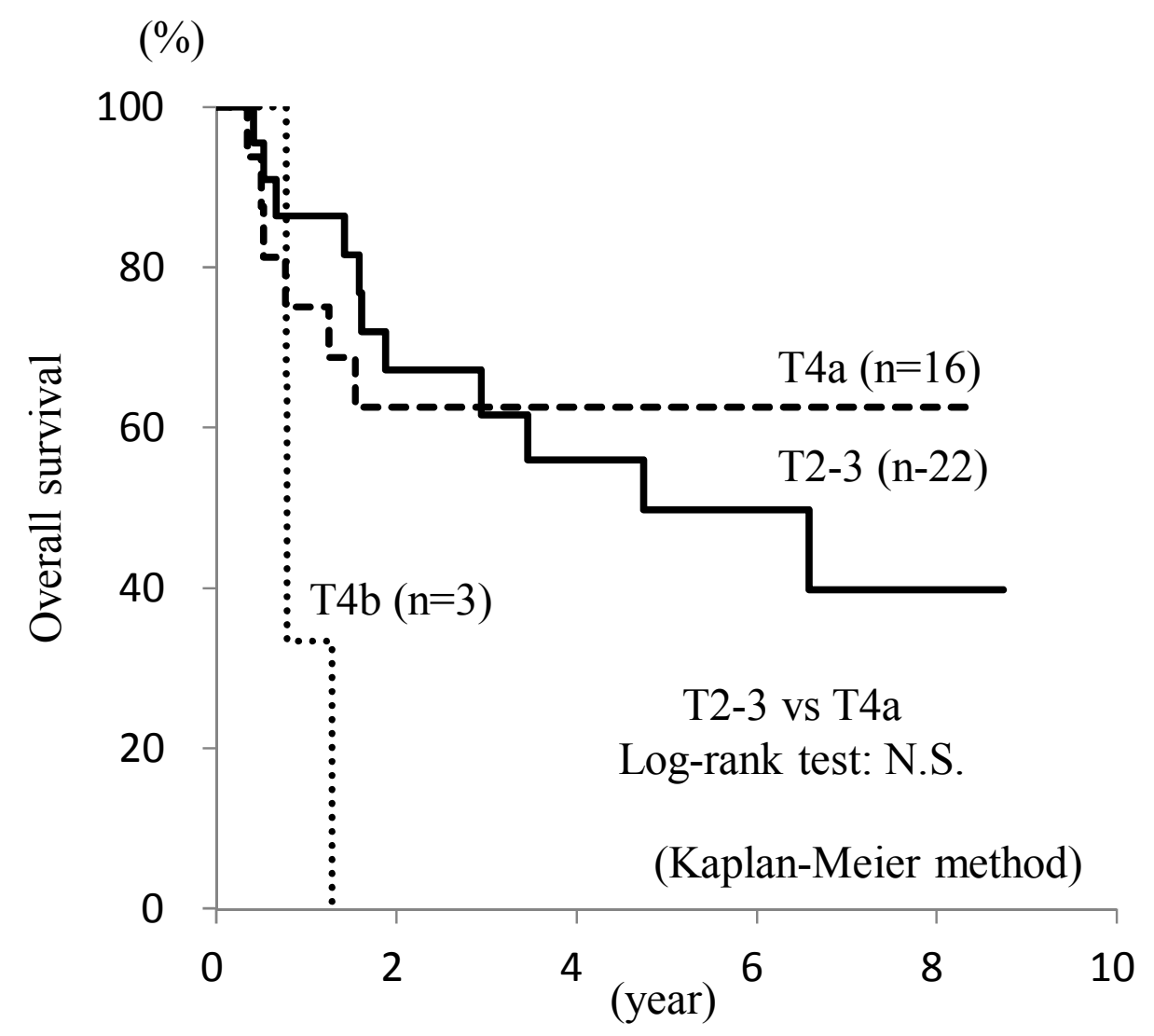

Patients at risk

$\begin{array}{lrrrrrrrrr}\text { T2-3 } & 22 & 19 & 14 & 11 & 10 & 7 & 7 & 4 & 1 \\ \text { T4a } & 16 & 12 & 10 & 10 & 9 & 6 & 3 & 3 & 2 \\ \text { T4b } & 3 & 1 & & & & & & & \end{array}$

Fig. 4B Overall survival (Unilateral versus Bilateral)

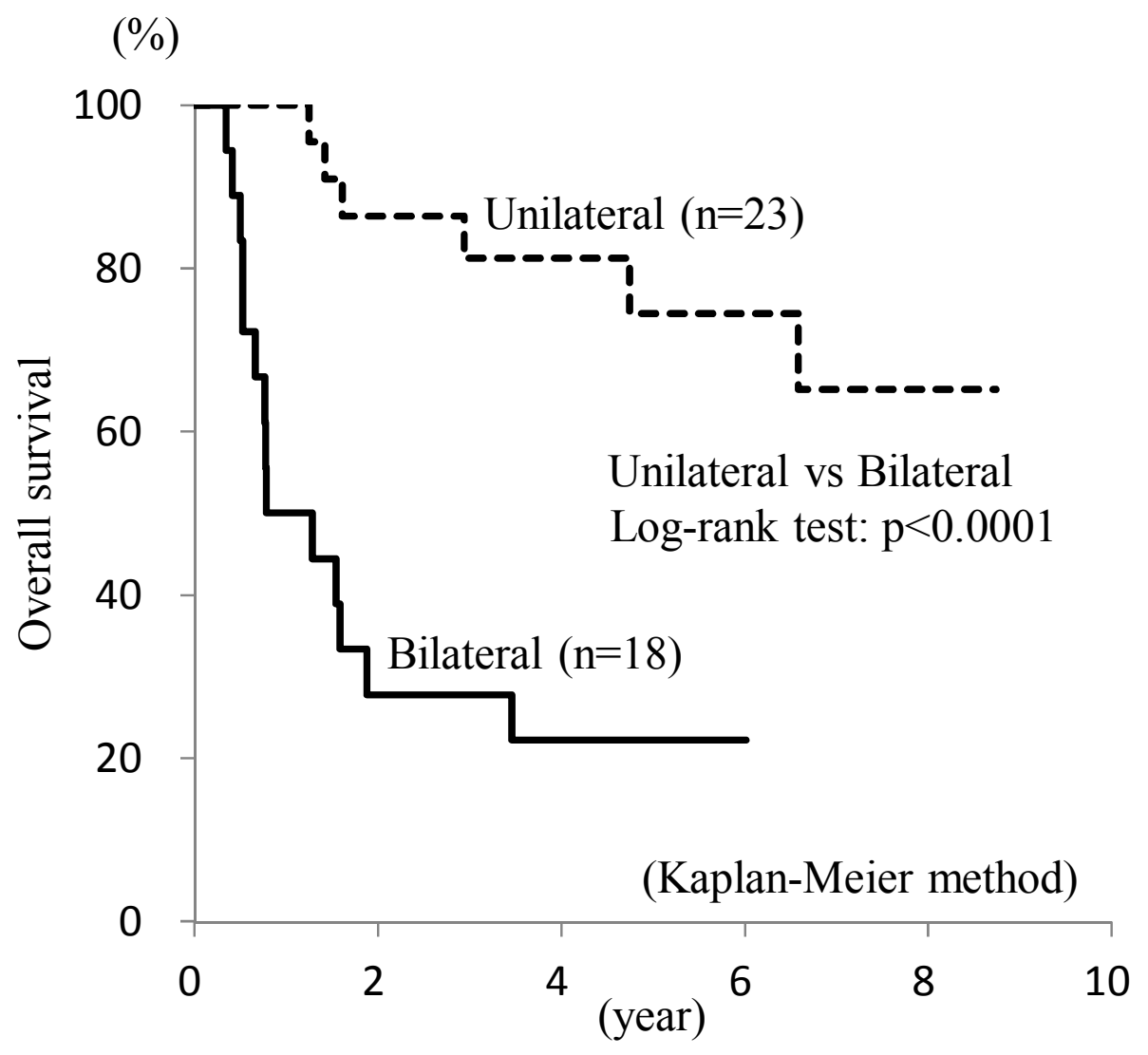

Patients at risk

$\begin{array}{llllllllll}\text { Unilateral } & 23 & 23 & 19 & 16 & 15 & 10 & 9 & 7 & 3\end{array}$

$\begin{array}{llllllll}\text { Bilateral } & 18 & 9 & 5 & 5 & 4 & 3 & 1\end{array}$ 
Fig. 5 Response to RADPLAT: Neck disease

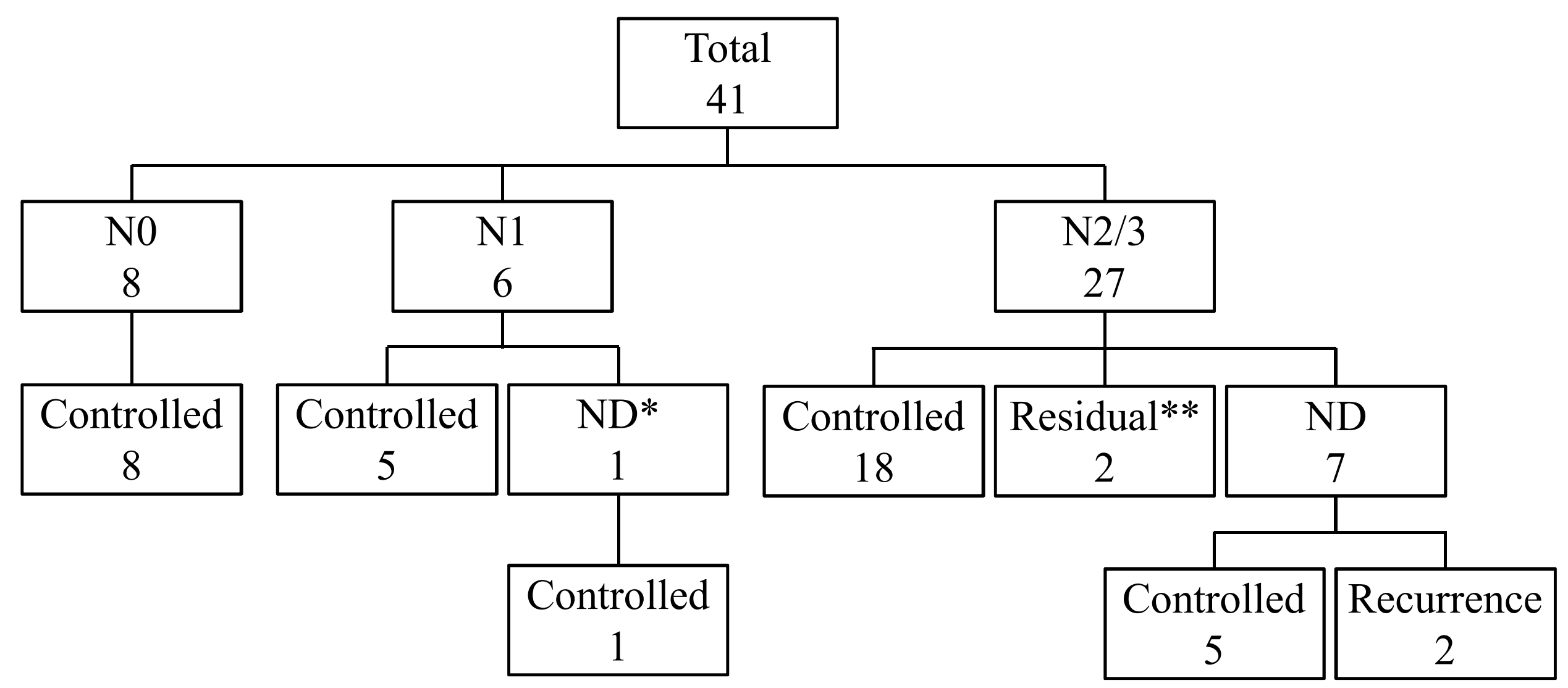

